NOTA TECNICA

\title{
Análisis comparativo de cinco métodos para la estimación de precipitaciones areales anuales en períodos extremos
}

\author{
Comparison analyses of five mean areal rainfall estimation methods in widely varying years \\ ROBERTO PIZARRO T. ${ }^{1}$, CLAUDIO RAMIREZ B. ${ }^{2}$, JUAN PABLO FLORES V ${ }^{3}$. \\ ${ }^{1}$ Depto. Gestión Forestal y Ambiental, Universidad de Talca, Casilla 721, Talca. Chile. \\ E-mail: rpizarro@utalca.cl \\ ${ }^{2}$ Dirección General de Aguas, Región del Maule, Chile. \\ E-mail: claudio.ramirez@moptt.gov.cl \\ ${ }^{3}$ Ingeniero Forestal. E-mail: jfloresv@utalca.cl
}

\begin{abstract}
SUMMARY
This study approaches the problems of mean areal rainfall estimation in three longitudinal sectors of the VII Region of Chile: the Andes mountain range, the central valley, and coastal mountain range. The methods used were the Mean Arithmetic (MA), Thiessen's Polygons (Th), Isohyets (Iso), Modified Thiessen (ThM) and Inverse Squared Distance (IDC). These methods were applied to two extreme series of pluvial data, dry (A.S.) and wet years (A.H.). An analysis and comparison of the methods recommended the application of any of the five methods for the central valley, but considered the MA method best due to its simplicity. A similar situation was found for the coastal mountain range, except the ThM. In the case of the Andes mountain chain, the only method not recommended was the IDC.
\end{abstract}

Key words: areal rainfall, dry and wet years, pluviometric study, method and error of estimation.

\section{RESUMEN}

El presente estudio aborda la problemática de la estimación de precipitaciones areales medias en tres sectores longitudinales de la VII Región de Chile (Cordillera de los Andes, Valle Central y Cordillera de la Costa). Las estimaciones se efectuaron a través de los siguientes métodos; Media Aritmética (MA), Polígonos de Thiessen (Th), Isoyetas (Iso), Thiessen Modificado (ThM) e Inverso de la Distancia al Cuadrado (IDC), los que fueron aplicados en dos series extremas de datos pluviales, años secos y húmedos. El análisis y comparación de los métodos aconseja la aplicación de cualquiera de ellos para el Valle Central, considerando de antemano la simplicidad de aplicación que manifiesta la Media Aritmética (MA). En la Cordillera de la Costa la situación es similar a la del Valle Central, a excepción de Thiessen Modificado (ThM). Para el caso de la Cordillera de los Andes, el único de los métodos estimadores que no se aconseja emplear es el Inverso de la Distancia al Cuadrado (IDC).

Palabras claves: precipitación areal, años secos y húmedos, pluviometría, métodos y errores de medición. 


\section{INTRODUCCION}

La relevancia del clima como factor regulador del medio natural y su incidencia sobre el ser humano, tanto de manera poblacional como sobre sus actividades, es innegable. En este sentido, la precipitación, al igual que la temperatura, es uno de los elementos climáticos que más influye sobre la naturaleza y su configuración. Su distribución temporal y espacial condiciona los ciclos agrícolas y forestales, así como el desarrollo de las principales especies vegetales y animales. Esta variable también influye, notoriamente, sobre otros rubros de la economía y la correspondiente producción de bienes y servicios, especialmente cuando es escasa.

Según UNESCO (1982), a pesar de la importancia de la precipitación en el balance hídrico, ésta no puede ser estimada con exactitud, ya que su evaluación se ve afectada por el error en la medida puntual (por ejemplo, funcionamiento del instrumento) y el error en la evaluación espacial de la precipitación caída sobre una gran superficie, a partir de valores puntuales (relacionado a la red de observación). Por consiguiente, la problemática del conocimiento de las cantidades precipitadas y su análisis espacial es de inmensa importancia, ya que trata de establecer las relaciones existentes entre los valores puntuales, registrados en diferentes estaciones, y los factores que los determinan. Esto se consigue a través de métodos de distribución espacial, permitiendo así extrapolar la información puntual de una estación meteorológica a un espacio más extenso, con el fin de obtener la precipitación de aquella superficie.

Los métodos para estimar las precipitaciones medias areales (M.E.P.M.A.) utilizados en esta investigación son la Media Aritmética, los Polígonos de Thiessen, las Isoyetas, Thiessen Modificado e Inverso de la Distancia al Cuadrado.

La Media Aritmética (MA) consiste en calcular el promedio de los datos pluviométricos puntuales de estaciones ubicadas en un área geográfica determinada (Linsley et al. 1988, MOP 1992, Sheng 1992, Llamas 1993, Pizarro 1993, Chow et al. 1994). La principal carencia del método es no considerar características anexas que pueden influir en las precipitaciones, como podría ser la presencia de cordones montañosos, cercanía a océanos, condiciones atmosféricas predominantes $\mathrm{u}$ otras.
Los Polígonos de Thiessen (Th) no presentan mayores complicaciones en su aplicación. Además de la información pluviométrica, requiere para su ejecución sólo de la ubicación espacial de las estaciones. No requiere de una inversión excesiva de tiempo, ni de materiales. A cada estación se le asigna una superficie, la cual es obtenida representando las estaciones en un plano y uniéndolas a través de rectas. A estas rectas posteriormente se les trazan sus mediatrices hasta que se intersectan entre sí. Con los límites del área en estudio y los que definen las mediatrices se obtiene la superficie de influencia para cada estación (Linsley et al. 1988, MOP 1992, Sheng 1992, Llamas 1993, Pizarro 1993, Chow et al. 1994, Fernández 1995, Lynch y Schulze 1997). La principal falencia del método radica en que sólo considera el posicionamiento de las estaciones y una superficie plana de influencia para cada una, sin considerar las diferencias topográficas que se pueden presentar. Además, asume que la precipitación de la estación es la misma de la zona que representa geométricamente, lo cual no siempre es cierto. Sin embargo, al ponderar cada estación por diferentes superficies, hipotéticamente sus resultados deberían ser más precisos que el método MA.

El Método de las Isoyetas (Iso) presenta más laboriosidad, tanto en tiempo como en recursos materiales. Para su ejecución es necesario disponer, además de las mediciones de precipitación en los períodos de interés y de las ubicaciones de los puntos de observación, de las precipitaciones normales de cada estación, del material cartográfico correspondiente y de un conocimiento espacial y atmosférico lo más detallado posible de las áreas consideradas. Al incorporar factores de la zona y de las precipitaciones, debería ser el mejor de los cinco métodos, pero a la vez tiene un alto grado de subjetividad dado por la experiencia y acceso a la información que posea el técnico encargado de la construcción de estas curvas.

El método denominado Thiessen Modificado (ThM) es una mezcla de los polígonos de Thiessen y las Isoyetas, y debiera presentar resultados bastante buenos. Entre la información que necesita para su puesta en práctica, además de la de Th, está la existencia previa de isoyetas. Este requisito condiciona bastante a ThM, debido a que su exactitud dependerá fuertemente de la calidad de esta información pluvial. Este método se basa en los dos últimos métodos descritos. Un inconveniente 
de ThM, al igual que Th, es que una variación en la cantidad o ubicación de las estaciones conlleva la realización de una nueva representación gráfica $\mathrm{y}$, por ende, a actualizar las mediciones de los polígonos, con los inconvenientes y nuevos cálculos asociados que ello demanda.

Finalmente, el método llamado Inverso de la Distancia al Cuadrado (IDC) es el más reciente entre los que se han analizado, y su importancia radica en la fácil aplicación en un S.I.G. El método IDC sería similar a MA, pero con el agregado de una ponderación por distancia. Dicha ponderación, al tener un factor cuadrático, recibe una influencia bastante fuerte del monto pluvial de las estaciones más cercanas y al considerar una serie o variedad de puntos de estimación puede formar agrupaciones concéntricas de los montos estimados en torno a las estaciones. Este método divide la cuenca o área de estudio en celdas, considerando que la precipitación dentro de una celda sin medida es una función de la precipitación de las celdas próximas que poseen medidas y del inverso de la distancia que las separa, elevada al cuadrado (MOP 1992, Chow et al. 1994, Lynch y Schulze 1997, Lynch 1998).

En este marco, la presente investigación pretende contribuir al análisis y evaluación de distintos métodos para la estimación de precipitaciones medias areales para períodos de años secos y húmedos, los que se aplicaron en tres áreas geográficas de la VII Región de Chile: Precordillera de los Andes, Valle Central y Cordillera de la Costa.

\section{MATERIAL Y METODOS}

Area de estudio: el área de aplicación de los métodos se encuentra en la VII Región administrativa del país, o Región del Maule, la cual posee una superficie de $30.535 \mathrm{~km}^{2}$ (figura 1).

El clima en la zona central del país, Aconcagua al Bío-Bío, es de tipo mediterráneo. En las precipitaciones hay un notorio aumento en los promedios anuales de norte a sur, siendo este valor en Talca cercano a los $700 \mathrm{~mm}$ anuales. De acuerdo al Balance Hídrico de Chile y sus estimaciones (1951-1980), la VII Región tiene una precipitación anual de $1.377 \mathrm{~mm}$, de los cuales escurren y se evaporan aproximadamente el $57 \%$ y $43 \%$, respectivamente. Según Pizarro (1999), la Cuenca del Maule $\left(20,9 \mathrm{mil} \mathrm{km}^{2}\right)$ tiene una precipitación media de $1.471 \mathrm{~mm} / \mathrm{año}$, de los que escurre el 58,5\%.

Selección de las estaciones pluviométricas: la selección de las estaciones se realizó de acuerdo a los siguientes criterios:

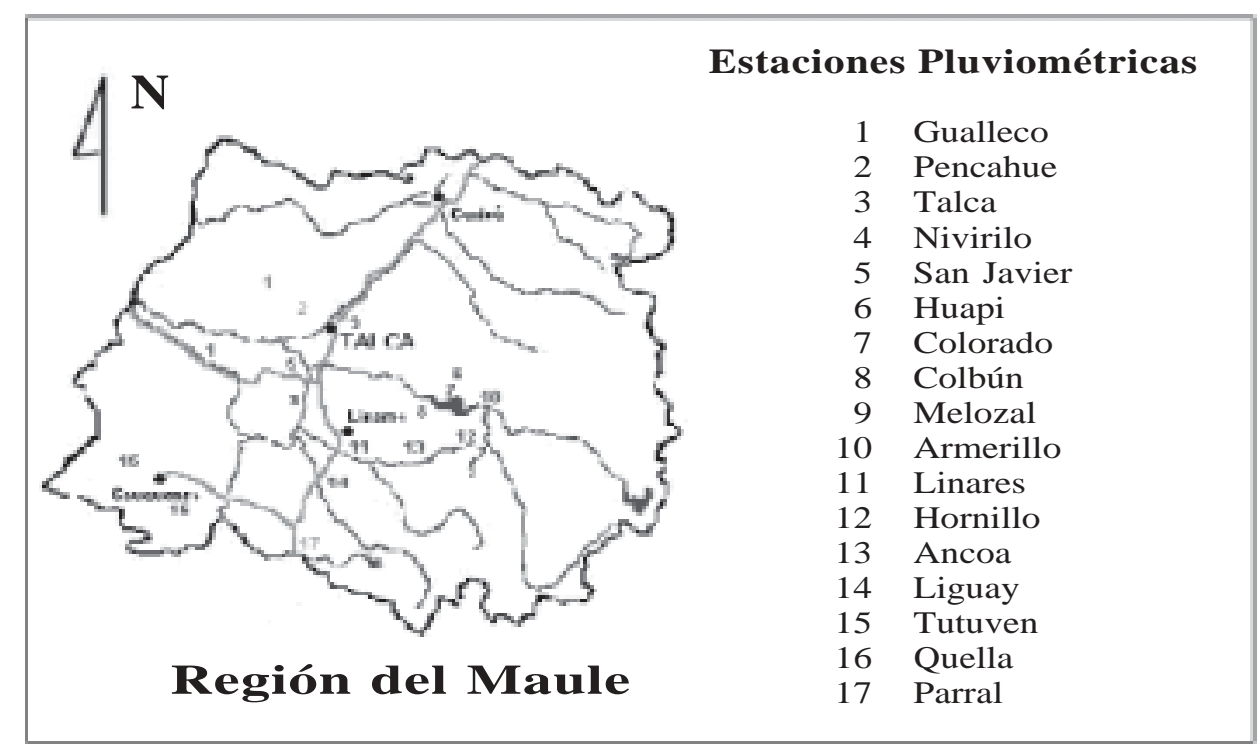

Figura 1. Mapa de ubicación de la Región del Maule. Location map of the Maule region of Chile. 
i) Mejor ubicación o más diferenciadas para cada una de las zonas geográficas.

ii) Las que, en conjunto, presenten una mayor densidad por zona.

iii) Las que presenten mayor número de años con registros confiables o mensualmente más completos, privilegiando a las que presenten más datos recientes, dado que deberían ser más exactos.

Un requisito importante en una preselección de estaciones es que éstas tengan un registro de datos de a lo menos 20 años. Dado que del total de estaciones pluviométricas de la Dirección General de Aguas (DGA) que existen en la Región del Maule (40) menos de 25 tienen un registro superior a 20 años, en esta etapa se seleccionaron 17. Posteriormente, las empleadas en el cálculo de las precipitaciones areales medias, luego de haber delimitado las áreas de estudio, fueron 14 estaciones.

Según la Organización Meteorológica Mundial (O.M.M.), para la existencia de una buena red pluviométrica debiera existir, en terrenos montañosos, a lo menos un pluviómetro cada $25 \mathrm{~km}^{2}$ y, en terrenos planos, uno cada $50 \mathrm{~km}^{2}$ (Pizarro 1993). Lo anterior contrasta al compararlo con la situación nacional, en particular la VII Región. Si se considera la existencia de una superficie regional de aproximadamente $30.000 \mathrm{~km}^{2}$ y alrededor de 40 estaciones estatales, da una densidad de una estación pluvial cada $750 \mathrm{~km}^{2}$, lo que manifiesta una notoria diferencia a lo propuesto por la O.M.M. Sin embargo, se debe tener en consideración que la cantidad de estaciones existentes en las zonas longitudinales definidas sería la adecuada para la Cordillera de la Costa y Valle Central, con un error admisible del $10 \%$. Análogamente, esto no es así para los Andes, donde el número de estaciones debería ser mayor (cuadro 1).

Procesamiento de la información: los datos obtenidos corresponden a valores mensuales, con su total anual por estación, a los cuales se les efectuó un manejo preliminar. Dicha actividad se realizó para el mayor número de estaciones posibles (17), debido a que aunque algunas de éstas no fuera considerada posteriormente en el cálculo de las precipitaciones medias areales, sí se emplearía en el método de las Razones Promedio para la completación de datos de estaciones próximas (Pizarro 1996, Lucero 1997), mientras que la corrección de los datos se llevó cabo por el método de las Curvas Doble Acumuladas o Doble Masa (UNESCO 1982, Linsley et al. 1988, Pizarro 1993).

Determinación del área de estudio para cada zona geográfica: para la obtención de las áreas en las cuales se aplicarían los M.E.P.M.A. se asignaron radios de influencia a la precipitación puntual. En otras palabras, se consideraron diferentes superficies de validez al dato pluviométrico puntual de una estación. Específicamente, se asignaron radios entre $5 \mathrm{~km}$ y $8 \mathrm{~km}$ a cada una de las estaciones seleccionadas, para así obtener una superficie circundante (menor a $200 \mathrm{~km}^{2}$ ) (figura 2).

\section{CUADRO 1}

Resumen de estaciones pluviométricas y diferenciación de años secos y húmedos. Rainfall station information and dry/wet years selected.

\begin{tabular}{|c|c|c|c|c|c|c|}
\hline Zonas geográficas & $\begin{array}{c}\mathrm{N}^{\mathrm{o}} \text { de } \\
\text { estaciones } \\
\text { seleccionadas }\end{array}$ & $\begin{array}{c}\mathrm{N}^{\mathrm{o}} \text { de } \\
\text { estaciones } \\
\text { utilizadas }\end{array}$ & $\begin{array}{l}\text { Superficie } \\
\left(\mathrm{km}^{2}\right)\end{array}$ & $\begin{array}{c}\mathrm{N}^{\mathrm{o}} \text { de } \\
\text { años } \\
\text { húmedos }\end{array}$ & $\begin{array}{l}\mathrm{N}^{\mathrm{o}} \text { de } \\
\text { años } \\
\text { secos }\end{array}$ & $\begin{array}{c}\mathrm{N}^{\mathrm{o}} \text { total } \\
\text { de } \\
\text { años }\end{array}$ \\
\hline C. de la Costa & 4 & 3 & 3.000 & 8 & 12 & 20 \\
\hline Valle Central & 7 & 5 & 2.250 & 9 & 12 & 21 \\
\hline C. de los Andes & 6 & 6 & 2.000 & 13 & 15 & 28 \\
\hline Total & 17 & 14 & 7.250 & 30 & 39 & 69 \\
\hline
\end{tabular}


Determinación del área de estudio

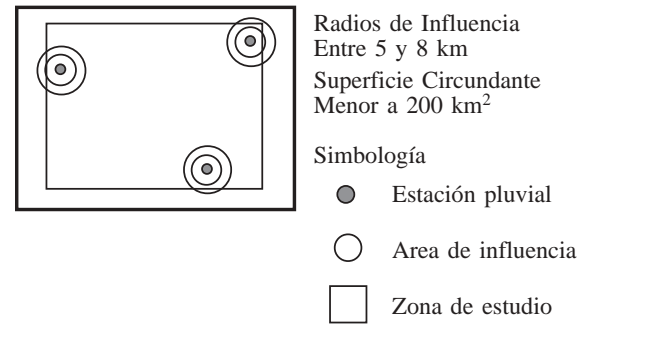

Figura 2. Delimitación de las áreas de estudio para la aplicación de los métodos.

Delimitation of study areas for the estimation methods.

Diferenciación en años secos y húmedos: para separar los datos en estas dos series extremas, se consideró el promedio normal de las precipitaciones para cada área geográfica, diferenciando como años secos aquellos que se encontraban con valores anuales bajo la precipitación normal y, años húmedos, los que presentaban valores superiores a ésta. Aunque generalmente los datos de precipitación son clasificados en tres o más subdivisiones, en este estudio sólo se consideraron dos subgrupos, debido a que, sin dejar de ser considerable la cantidad de registros, éstos no son los suficientes como para realizar más diferenciaciones (cuadro 1).

Cálculos de las precipitaciones medias areales: delimitadas las áreas y diferenciadas las series, se aplicaron los siguientes M.E.P.M.A.: Media Aritmética (MA), Método de los Polígonos de Thiessen (Th), Método de las Isoyetas (Iso), Método de Thiessen Modificado (ThM) y el Método del Inverso de la Distancia al Cuadrado (IDC) (cuadro 2).

Cabe considerar que las isoyetas se construyeron para años húmedos y secos. En el Valle Central fue por interpolación lineal, y en ambas cordilleras fueron obtenidas por interpolación, pero considerando el criterio de la variación de altura y apoyándose en el perfil pluviométrico, a través de un método de precipitación. Los ajustes realizados a diversas relaciones revelaron que el mejor resul-

\section{CUADRO 2}

Fórmulas: Métodos de estimación de precipitaciones areales.

Formulas: Estimation methods for areal rainfall.

\begin{tabular}{|c|c|c|}
\hline Media Aritmética (a) & Isoyetas (b) & Método Precipitación (c) \\
\hline $\begin{aligned} & \mathrm{n} \\
\mathrm{Pm} & =\sum \mathrm{Pi} / \mathrm{n} \\
\mathrm{i} & =1\end{aligned}$ & $\begin{array}{c}\sum_{\substack{n \\
\mathrm{i}=1}}((\mathrm{Pi}+1+\mathrm{Pi}) / 2) * \mathrm{~S} \mathrm{i}+1, \mathrm{i} \\
\mathrm{Pm}=\mathrm{St}\end{array}$ & $\mathrm{Pp}=\mathrm{a}+\mathrm{b} * \mathrm{H}^{\mathrm{z}}$ \\
\hline Thiessen (d) & Thiessen Modificado (e) & IDC (f) \\
\hline $\begin{array}{c}\mathrm{n} \\
\sum \mathrm{Si} * \mathrm{Pi} \\
\mathrm{i}=1 \\
\mathrm{Pm}=1 \\
\mathrm{n} \\
\sum \mathrm{Si} \\
\mathrm{i}=1\end{array}$ & $\begin{aligned} \mathrm{Pm}= & \mathrm{n}(\mathrm{Pm} \text { ai } / \mathrm{Pm} \text { ei }) * \mathrm{P} \text { eai } *(\mathrm{Si} / \mathrm{St}) \\
& \mathrm{i}=1\end{aligned}$ & 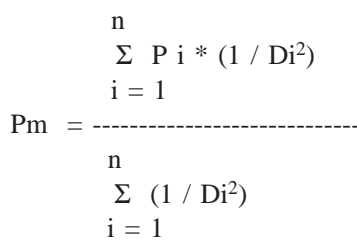 \\
\hline
\end{tabular}

SIMBOLOGIA

\footnotetext{
Pm : Precipitación media del área de estudio [mm].

n : Número de estaciones en análisis.

$\mathrm{Si}+1, \mathrm{i}:$ Superficie entre dos isoyetas consecutivas $\left[\mathrm{km}^{2}\right]$.

Pp : Precipitación [mm]

$\mathrm{H}$ : Altitud [ms.n.m.].

$\mathrm{Si} \quad$ : Superficie del área de influencia i $\left[\mathrm{km}^{2}\right]$.

$\mathrm{Di} \quad$ : Distancia entre estación y celda correspondiente $[\mathrm{km}]$

Pm ai : Precipitación media del área de influencia de la estación

según Thiessen, obtenida por isoyetas medias anuales [mm].
} 
tado es la relación Precipitación Normal vs Altitud. De esta manera se obtuvieron los coeficientes y constantes respectivas (ai, bi y zi).

Por otra parte, la aplicación del método Thiessen Modificado se realizó en función de las isoyetas correspondientes al "Balance Hídrico de Chile 1987” (MOP 1988).

Análisis estadístico: se caracterizó estadísticamente cada método para las tres áreas en estudio. A los métodos Media Aritmética (MA), Thiessen (Th), Thiessen Modificado (ThM) e Inverso de la Distancia al Cuadrado (IDC) se les realizaron gráficas de probabilidad normal. Adicionalmente, se realizaron Pruebas de Hipótesis de efectos fijos de tratamientos (métodos), cuya hipótesis nula (Ho) indica que los efectos de los métodos sobre la media son iguales $\mathrm{y}$, a la vez, toman el valor cero. Lo anterior se realiza a través de un análisis de varianza (Montgomery et al. 1996). A su vez, se desarrollaron pruebas de hipótesis de diferencias entre pares de medias de distribuciones normales con varianzas desconocidas y distintas, que compararon todos los pares de medias, donde la hipótesis nula (Ho) indica que las medias no presentan diferencias significativas. Asimismo, se aplicó el test de rangos múltiples de Duncan para encontrar diferencias entre una serie de medias. Aquí se prueban todos los pares en una aplicación del test. Lo anterior se llevó a cabo para los datos de años secos y húmedos y para cada zona geográfica.

\section{RESULTADOS Y DISCUSION}

En primer lugar, la acumulación de los valores medios anuales se ajustan bastante bien a una distribución normal $\mathrm{N}(0, \sigma)$, pudiendo caracterizar a cada método con una expresión matemática de parámetros definidos y conocidos. En este sentido (cuadro 3), los valores de las precipitaciones medias areales por cada método, por zona y para los correspondientes períodos húmedos y secos, destacan que, en la Cordillera de la Costa, la situación es similar al Valle Central, a excepción de los resultados obtenidos con Thiessen Modificado (ThM). Cabe recordar que ésta fue el área geográfica de mayor extensión y menor número de estaciones, por lo cual también es aconsejable la utilización a futuro de los valores que vayan entregando las nuevas estaciones. Asimismo, en áreas don- de los montos precipitados no son muy excesivos, la existencia de isoyetas cada $100 \mathrm{~mm}$ no es la óptima, como aconteció en gran parte de la Cordillera de la Costa.

Por otra parte, se puede destacar que en el Valle Central la aplicación de cualquiera de los cinco métodos es válida. Por consiguiente, y considerando de antemano la simplicidad y facilidad de aplicación, se aconseja el uso de la Media Aritmética (MA). Lo anterior se debería principalmente a la mayor homogeneidad orográfica que presenta esta parte del territorio regional.

En cuanto a la Cordillera de los Andes, el test de hipótesis de diferencias entre pares de medias evidenció diferencias significativas sólo para el método IDC. En este caso, sería importante considerar otro factor de ponderación para dicho método, relacionado igualmente con la distancia o sumado a esto un factor de altitud o direccionalidad de la pendiente (exposición), debido a que la variabilidad que presentan las precipitaciones, en pocos kilómetros, en las zonas más altas de los Andes, es realmente notable en comparación a la de sectores más planos.

En cuanto a la comparación de los cinco métodos es importante destacar la fuerte semejanza existente entre los resultados asociados a los métodos MA y Th, siendo éstos los únicos que nunca presentaron diferencias entre sus medias. En la aplicación de los métodos se debe considerar la facilidad y rapidez que presenta MA en relación al resto, por lo cual se puede aconsejar su utilización cuando los puntos de medición se distribuyan de forma uniforme sobre el área.

Caso análogo al anterior presenta Th, principalmente en terrenos no montañosos. El planteamiento de este método asigna la precipitación de la estación más cercana a los puntos dentro de cada polígono, por lo que hipotéticamente debería ser más exacto que MA. Pero Th presenta el inconveniente de no poseer flexibilidad, al ser necesario construir una nueva serie de polígonos cada vez que se modifique la red de estaciones existentes, así como en el caso de no existir mediciones en alguna de las estaciones para un período específico, ni puedan ser éstas completadas satisfactoriamente.

IDC fue el método que manifestó las mayores diferencias, principalmente en la zona andina. Por ello, no se debe dejar de considerar que depende fuertemente de una alta densidad de puntos de 


\section{CUADRO 3}

Precipitaciones areales anuales medias.

Mean areal rainfall.

\begin{tabular}{|c|c|c|c|c|c|c|}
\hline \multicolumn{2}{|c|}{ Métodos } & MA & Th & Iso & ThM & IDC \\
\hline Zona & Años & (mm/año) & (mm/año) & (mm/año) & (mm/año) & (mm/año) \\
\hline C. Costa & $\begin{array}{c}\text { Húmedos } \\
\text { Secos }\end{array}$ & $\begin{array}{r}1.109,1 \\
688,4\end{array}$ & $\begin{array}{r}1.127,8 \\
700,9\end{array}$ & $\begin{array}{r}1.134,2 \\
708,7\end{array}$ & $\begin{array}{r}1.009,5 \\
626,6\end{array}$ & $\begin{array}{r}1.139,1 \\
719,3\end{array}$ \\
\hline Valle Central & $\begin{array}{c}\text { Húmedos } \\
\text { Secos }\end{array}$ & $\begin{array}{r}1.028,3 \\
673,3\end{array}$ & $\begin{array}{r}1.017,3 \\
659,9\end{array}$ & $\begin{array}{r}1.013,0 \\
673,0\end{array}$ & $\begin{array}{r}1.015,6 \\
656,8\end{array}$ & $\begin{array}{r}1.014,3 \\
630,5\end{array}$ \\
\hline C. Andes & $\begin{array}{c}\text { Húmedos } \\
\text { Secos }\end{array}$ & $\begin{array}{l}2.047,0 \\
1.303,2\end{array}$ & $\begin{array}{l}2.078,5 \\
1.328,6\end{array}$ & $\begin{array}{l}1.940,1 \\
1.273,5\end{array}$ & $\begin{array}{l}2.098,0 \\
1.346,2\end{array}$ & $\begin{array}{l}1.677,1 \\
1.065,4\end{array}$ \\
\hline
\end{tabular}

medición, sobre todo si el terreno es poco homogéneo. Además, se debe considerar que en un sector como la Cordillera de los Andes las estaciones pueden estar ubicadas en lugares no muy representativos de los niveles pluviales existentes.

\section{CONCLUSIONES}

La utilización de los M.E.P.M.A. presenta diferencias, tanto en lo relacionado a la complejidad y laboriosidad de cada uno en particular, como en los requisitos necesarios para su aplicación. Adicionalmente, se estableció que existen diferencias entre los distintos M.E.P.M.A. aplicados, dentro de algunas zonas de la VII Región, asociadas éstas a sus posiciones longitudinales y su respectiva situación orográfica y altitudinal.

En el caso de la Cordillera de la Costa, para años secos no existen grandes diferencias significativas entre los distintos métodos y la variación porcentual máxima la representa el método de Thiessen Modificado (ThM). Por otra parte, en años húmedos se manifiesta una leve diferencia entre ThM y la totalidad del resto de los métodos en análisis. Por lo tanto, no es aconsejable la estimación de las precipitaciones areales en años secos y húmedos, para la Cordillera de la Costa, empleando el método de ThM. Para el Valle Central, en años secos no existen diferencias significativas entre los métodos y la mayor diferencia porcentual la presenta IDC. De igual forma, en años húmedos no se vislumbran diferencias entre los métodos. Por consiguiente, se concluye que para el Valle Central en años secos y húmedos la aplicación de cualquiera de los cinco métodos es relativamente indiferente en cuanto a sus resultados. En la Cordillera de los Andes, para años secos sí existen diferencias significativas entre los métodos, originadas por IDC. Para el caso de los años húmedos, sí se presentan diferencias significativas entre IDC y todos los demás métodos, por lo cual se concluye que este método manifiesta deficiencias en esta zona geográfica.

Como guía para nuevas investigaciones, donde sea necesario disponer de los montos precipitados sobre una determinada superficie, y considerando los métodos analizados, se puede recomendar que la selección del método más adecuado debe tener en consideración la distribución espacial y temporal de la precipitación, la densidad y distribución de la red de medición en función de la zona geográfica, la disponibilidad de datos (incluye cantidad y calidad) y la posibilidad de ejecución práctica del método.

Finalmente, y de acuerdo al estudio realizado, el método más recomendable para la VII Región es Polígonos de Thiessen (Th), ya que en este análisis comparativo manifestó resultados bastantes aceptables dentro de la generalidad de situaciones, al igual que la Media Aritmética, pero Th además agrega en su formulación un factor externo relacionado a la superficie adyacente a cada estación. 
BOSQUE 24(3): 31-38, 2003

Análisis comparativo de cinco métodos para la estimación de precipitaciones areales anuales..

\section{BIBLIOGRAFIA}

CHOW, V., D. MAIDMENT, L. MAYS. 1994. Hidrología Aplicada. Editorial Mc Graw-Hill. Bogotá, Colombia. 583 p.

FERNANDEZ, F. 1995. Manual de Climatología Aplicada. Clima, medio ambiente y planificación. Editorial Síntesis S. A., Madrid, España. 285 p.

LINSLEY, R., M. KOHLER, J. PAULHUS. 1988. Hidrología para Ingenieros. $2^{\mathrm{a}}$ Ed. Editorial Mc Graw-Hill Latinoamericana, D. F. México. 386 p.

LLAMAS, J. 1993. Hidrología General. Principios y Aplicaciones. Editorial Universitaria del País Vasco, Bilbao, España. $635 \mathrm{p}$.

LUCERO, A. 1997. Análisis Probabilístico Espacial y Temporal de las Precipitaciones Pluviales Anuales de la VII Re gión. Escuela de Ingeniería Forestal, Facultad de Recursos Naturales, Universidad de Talca, Talca, Chile. 69 p.

LYNCH, S. 1998. Converting Point Estimates of Daily Rainfall Onto a Rectangular Grid. Department of Agricultural Engineering, University of Natal South Africa. www. geocomputation.org/1998/93/gc_93.htm. Internet: Marzo, 1998.

LYNCH, S., R. SCHULZE. 1997. Techniques for estimating areal daily rainfall. Department of Agricultural Engineering, University of Natal South Africa http://gis esri.com/library/ userconf/proc95/to250/p241.html. Internet: Enero, 1998.
MONTGOMERY, D., G. RUNGER, 1996. Probabilidad y Estadística Aplicadas a la Ingeniería. Mc Graw-Hill Latinoamericana, D. F. México. 895 p.

MOP, DGA, Chile. 1988. Balance Hídrico de Chile - 1987. Dirección General de Aguas. Ministerio de Obras Públicas, Santiago, Chile. 24 p.

PIZARRO, R. 1993. Elementos Técnicos de Hidrología III. Proyecto Regional Mayor sobre Uso y Conservación de Recursos Hídricos en Areas Rurales de América Latina y el Caribe. Universidad de Talca, Talca, Chile. 135 p.

PIZARRO, R. 1996. Análisis Comparativo de Métodos Matemáticos Precipitación - Escorrentía en Cuencas de la España Peninsular. Departamento de Ing. Forestal, Escuela Técnico Superior de Ingeniería de Montes, Univ. Politécnica de Madrid, Madrid, España. 277 p.

PIZARRO, R. 1999. En Informe País: Estado del Medio Ambiente en Chile - 1999. Capítulo 2: Recursos Hídricos. Gobierno de Chile. Primera edición: 77-129.

SHENG, T. 1992. Manual de Campo para la Ordenación de Cuencas Hidrográficas: Estudio y Planificación de Cuencas Hidrográficas. Guía FAO: Conservación 13/6. Roma, Italia. $185 \mathrm{p}$

UNESCO, ROSTLAC. 1982. Guía Metodológica para la Elaboración del Balance Hídrico de América del Sur. Oficina Regional Ciencia y Tecnología, Unesco para América Latina y el Caribe, Montevideo, Uruguay. 130 p. 